NOTA DEL DIRECTOR: Al ser la mano un órgano exploratorio, los encuentros desagradables con objetos punzantes biológicos no son infrecuentes. Sin dudar que la etiología en el caso anterior pueda ser el veneno, y no el aguijón que hubiera entrado en el canal digital, me ha parecido apropiado ampliar este artículo con una visión más global del problema por parte del Dr Martínez-Taboada especialista en Reumatología. Los reumatólogos están más familiarizados con el problema de monoartritis o tenosinovitis sin filiar que vagan de un sitio a otro sin diagnóstico, y cuya causa última es ... «esa palmera, o ese erizo con el que me pinché hace 1 año». El artículo del Dr Martínez Villén y colaboradores es un reflejo de la importancia de esta patología para el cirujano de mano.

\title{
Sinovitis por cuerpo extraño (Editorial invitada)
}

\author{
V. M. Martínez-TABoAda \\ Correspondencia: \\ Dr. V. M. Martínez-Taboada \\ Médico Adjunto. Servicio de Reumatología \\ Hospital Universitario «Marqués de Valdecilla» \\ Avda. De Valdecilla S/N. Santander 39008. España \\ e-mail: vmartinezt@medynet.com
}

Servicio de Reumatología. Hospital Universitario «Marqués de Valdecilla». Santander. España.

\begin{abstract}
e denomina sinovitis por cuerpo extraño (SCE) a la inducida por la penetración de partículas de diversa naturaleza en el interior o en la vecindad de la sinovial que recubre las articulaciones o las vainas tendinosas ${ }^{1}$. Aunque se considera que este tipo de patología es mucho más frecuente de lo que se piensa habitualmente, no existe ningún estudio sobre la incidencia y prevalencia de la SCE, y toda la información de la que disponemos actualmente esta basada en la publicación de numerosos casos aislados y pequeñas series de pacientes ${ }^{2-6}$.

Los cuerpos extraños más frecuentes son las espinas vegetales y animales, aunque otro tipo de agentes como metales, cristal o sustancias plásticas, también deben ser considerados en este tipo de patología. De igual modo, pequeños fragmentos de metal, silicona, metacrilato, polietileno u otros materiales procedentes de prótesis articulares pueden ser los responsables de una sinovitis granulomatosa que se comporta de igual forma que la clásica $\mathrm{SCE}^{7}$. Más recientemente, el uso de determinados agentes utiliza-
\end{abstract}

dos de forma local para el tratamiento de la artrosis (ácido hialurónico) también se ha asociado con el desarrollo de sinovitis granulomatosa indistinguible de la $\mathrm{SCE}^{8}$. Siendo las espinas vegetales y animales (especialmente de animales marinos) los agentes etiológicos más frecuentes, es lógico asumir que este tipo de patología va a ocurrir con mayor frecuencia en profesionales o individuos aficionados a la jardinería, agricultores, pescadores o manipuladores de pescado. Ante la presencia de una sinovitis localizada de curso subagudo o crónico en estos grupos de riesgo, la anamnesis es clave para sospechar la posibilidad de una SCE. Una situación algo más compleja es cuando la SCE se produce en niños que suelen estar expuestos a punciones accidentales, y en los que la anamnesis suele ser a menudo menos contributoria.

Aunque la mayoría de los pacientes va a recordar la lesión punzante inicial, existe un porcentaje no despreciable de los pacientes que no recuerda o no relaciona el trauma inicial con el desarrollo de la sinovitis. Tras un periodo in- 
tercrítico variable, el paciente desarrolla una sinovitis crónica. Como es lógico, las articulaciones más frecuentemente afectadas son las más expuestas, especialmente las articulaciones de las manos (sobre todo las interfalángicas) y en el caso de los niños son también especialmente frecuentes las rodillas. La sintomatología referida por el paciente, el grado de sinovitis y la limitación de la capacidad funcional va a ser también muy variable. La sintomatología sistémica no suele aparecer salvo en el caso de algunas SCE por espinas animales que puedan contener determinadas sustancias tóxicas.

Del mismo modo que no suelen aparecer manifestaciones sistémicas tampoco suelen aparecer alteraciones analíticas reseñables. El estudio del líquido sinovial suele mostrar un líquido inflamatorio, con predominio de polimorfonucleares, siendo excepcional la visualización del cuerpo extraño en el líquido sinovial.9 Es fundamental en este tipo de procesos el realizar cultivos tanto del líquido sinovial como de la membrana sinovial obtenida durante la sinovectomía con objeto de eliminar la posibilidad de una artritis séptica concomitante. En pacientes que presentan este tipo de lesión tras exposición al medio marino, es especialmente recomendable el realizar también cultivos para micobacterias. En el diagnóstico de la SCE va a ser clave la anamnesis, especialmente si el paciente recuerda el antecedente de la punción o bien si pertenece a un grupo de riesgo. Desde el punto de vista de la imagen, la radiología simple es sólo de utilidad en los casos de cuerpos extraños radiodensos y de suficiente tamaño. Aunque no existe ningún estudio bien diseñado al respecto, se ha sugerido que tanto la ecografía, como el TAC o la RMN pueden ser de utilidad a la hora de identificar cuerpos extraños. ${ }^{10}$ En cualquier caso, el diagnóstico suele requerir una biopsia sinovial cuyo estudio histológico, microbiológico, y la identificación del cuerpo extraño proporcionan el diagnóstico definitivo. Ocasionalmente puede no identificarse el cuerpo extraño en las muestras obtenidas tras la sinovectomía, pero la historia de una punción previa, un cuadro clínico y un estudio histopatológico compatibles, pueden ser suficientes para el diagnóstico. Así mismo, se recomienda examinar las muestras de tejido al microscopio con luz polarizada con objeto de identificar fragmentos birrefringentes. ${ }^{1}$

Aproximadamente, en una tercera parte de los casos se produce una resolución espontánea de la SCE. ${ }^{1}$ Sin embargo, el tratamiento quirúrgico adecuado va a ser fundamental en la mayoría de los pacientes y se va a acompañar de una disminución en la duración y en la intensidad de la sintomatología. El tratamiento consiste en la realización de una sinovectomía extensa y lavado articular. ${ }^{9}$

En resumen, la SCE es una entidad probablemente mucho más frecuente de lo que se sospecha. En cualquier paciente con una monoartritis o tenosinovitis crónica en un área expuesta debe considerarse esta posibilidad. Para el diagnóstico será clave una anamnesis cuidadosa, aunque en la mayoría de los casos, este va a provenir del estudio histopatológico cuidadoso de la membrana sinovial resecada. La cirugía, consistente en una sinovectomía extensa, va a ser a menudo no sólo el elemento clave en el diagnóstico, sino el tratamiento definitivo en la mayoría de los pacientes. 


\section{BIBLIOGRAFÍA}

1. Fereiro Seoane, J. L.; Gomez Rodríguez, N.: Sinovitis por cuerpo extraño. En: Tratado de Reumatología 1998 (I); 112529. E. Pascual, V. RodríguezValverde, J. J. Gómez-Reino, y J. Carbonell, editores. Arán Ediciones SA.

2. Eidelman, M.; Bialik, V.; Miller, Y.; Kassis, I.: Plantar puncture wounds in children: analysis of 80 hospitalized patients and late sequelae. Isr Med Assoc J. 2003; 5: 268-71.

3. Kandel, L.; Friedman, A.; Chaimski, G.; Howard, C.; Mann, G.; Lowe, J.: Foreingbody synovitis mimicking septic arthritis of the knee. Arthroscopy 2001; 17: 993-6.

4. Miller, E. B.; Gilad, A.; Schatt- ner, A.: Cactus thorn arthritis: case report and review of the literature. Clin Rheumatol 2000; 19: 490-1.

5. Ajmal, N.; Nanney, L. B.; Wolfort, S. F.: Catfish spine envenomation: a case of delayed presentation. Wilderness Environ Med 2003; 14: 101-5.

6. Guyot-Drouot, M. H.; Rouneau, D.; Rolland, J. M. et al.: Arthritis, tenosynovitis, fasciitis, and bursitis due to sea urchin spines. A series of 12 cases in Reunion Island. Joint Bone Spine 2000; 67: 94-100.

7. Khoo, C. T.; Davison, J. A.; Ali, M.: Tissue reaction to titanium debris following Swanson arthroplasty in the hand: a report ot two cases. Hand Surg 2004; 29: 152-4.

8. Michou, L.; Job-Deslandre, C.; de Pinieux, G.; Kahan, A.: Granulomatous synovitis after intraarticular Hylan GF-20. A report ot two cases. Joint Bone Spine 2004; 71: 438-40.

9. Reginato, A. J.; Ferreiro, J. I.; O’Connor, C. R.; Barbazan, C.; Arasa, J.; Bednar, J.; Soler, J.: Clinical and pathological studies of twenty-six patients with penetrating foreing body injury to the joints, bursae, and tendon sheats. Arthritis Rheum 1990; 33: 1753-62.

10. Chan, M.; Chowchuen, P.; Workman, T. et al.: Silicone synovitis: MR imaging in five patients. Skeletal Radiol 1998; 27: 13-17. 